\title{
Dynamics, Information and Control in Physical Systems ${ }^{\star}$
}

\author{
Alexander L. Fradkov \\ Institute for Problems of Mechanical Engineering of RAS, \\ 61 Bolshoy Ave. V.O., St.Petersburg, 199178, Russia \\ alf@control.ipme.ru
}

\begin{abstract}
The subject and methodology of an emerging field related to physics, control theory and indormation theory are outlined. The paradigm of cybernetical physics as studying physical systems by cybernetical means is discussed. Examples of transformation laws describing excitability properties of dissipative and bistable systems are presented. A possibility of application to analysis and design of information transmission systems and complex networks is discussed.
\end{abstract}

\section{Introduction}

Modern communication networks are evolving very rapidly. Their element sizes are going down while signal rates are going up. Among different tendencies influencing development of the principles of the future generation communication networks the following two seem very important.

1. Complexity of systems and networks of the future requires very high speed of information exchange, close to physical limits. It makes conventional study of information flows in the network not sufficient. Networks should be treated as physical systems with their properties and limitations. Particularly, energy exchange flows should be taken into account. It means that information should be treated as a physical quantity, like energy or entropy. Quoting R. Landauer, "Information is tied to a physical representation and therefore to restrictions and possibilities related to laws of physics" 1].

An importance of understanding interrelations between energy exchange and information transmission was recognized still in the 1940s. The founder of the information theory C. Shannon derived in 1948 that at least $k T \ln 2$ units of energy is needed to transmit a unit (1 bit) of information in a linear channel with additive noise [2, where $T$ is absolute temperature and $k$ is the Boltzmann constant. This is just an energy required to make a signal distinguishable above the thermal background. In 1949 J. von Neumann extended that statement to the following principle: any computing device, natural or artificial must dissipate

\footnotetext{
* Supported by Russian Foundation of Basic Research (grant RFBR 02-01-00765) and Complex Program of the Presidium of RAS \#19 "Control of mechanical systems", project 1.4.
} 
at least $k T \ln 2$ energy per elementary transmission of 1 unit of information [3]. Next contribution into linking energy and information was made by R.Landauer: "Data processing operation has irreducible thermodynamic cost if and only if it is logically irreversible 4. It seemingly contradicts the von Neumann's principle. Besides, it is not clear how to transmit information with minimal energy.

A clarification was made again by Landauer who considered the case of nonlinear channels in which information is carried in the internal state of a material body (e.g. a bistable molecule with two states separated by a high-energy barrier). He established both for classical [5] and for quantum [6] case that a bit can be transmitted without minimal unavoidable cost. It means that a communication network with only a small loss of transmitted information may require only a small amount of energy for information transmission. However, the problem of organizing an efficient transmission is still to be solved, since the solution proposed by Landauer is a sort of existence theorem and does not suggest a way to design such a transmitter. For example, how to put a carrier of a bit of information into one or another well of a bistable potential? In fact here we face a control problem which is not easy to solve on a molecular level. And despite existence of publications arguing against Landauer's principle (see e.g. 7]) it is clear that there exist physical limits for information transmission and sophisticated methods are needed to approach them.

2. Complexity of future networks does not allow to perform their analysis and design in advance, before their functioning starts. Networks should be created as evolving and adaptive structures that are adjusted during their normal functioning. Although adaptive network concepts are well studied (see, e.g. 8, new challenges demand for fast adaptation which rate is comparable with the rate of information exchange in a network. Analysis and design of such structures are within the scope of the modern control theory. It means that future network analysis and design paradigms should heavily rely upon control theory and methods.

A consequence of the above observations is importance of deepening interaction between physics and control theory (in broad sense - cybernetics). However, although both physics and cybernetics were booming in the last century and made revolutionary contributions into science and technology, interaction between two sciences was almost negligible until recently. The reason lies, perhaps, in totally different methodologies of these sciences. Indeed, physics (particularly, mechanics) is a classical descriptive science and its aim is to describe the natural behavior of the system. The cybernetics (control science), in the contrary, is a paradigm of prescriptive science with the main aim to prescribe the desired behavior of the system [9]. In other words, the purpose of physics is to describe and analyze systems while the purpose of cybernetics is to transform systems by means of controlling action in order to achieve a prescribed behavior.

Surprisingly, the situation has changed in the beginning of the 1990s. New avenue of research in physics was opened by results in control and synchronization of chaos. It was discovered by E.Ott, C.Grebogi and J.Yorke [10] that even 
small feedback action can dramatically change behavior of a nonlinear system, e.g. turn chaotic motions into periodic ones and vice versa. The idea has become popular in the physics community almost instantaneously. Since 1990 hundreds of papers were published demonstrating ability of small control to change dynamics of the systems significantly, even qualitatively. By the end of the year 2000 the number of quotations of the paper [10] exceeded 1100 while the total number of the papers related to control of chaos exceeded 2500. The number of papers published in peer reviewed journals achieved 300-400 papers per year by the beginning of the XXIst century.

Similar picture can be observed in other fields of interaction between physics and cybernetics: control of quantum systems, control of lasers, control of plasma, beam control, control thermodynamics, etc. Reachability of the control goal by means of arbitrarily small control was observed in a broad class of oscillatory systems 11

It is important that the obtained results were interpreted as discovering new properties of physical systems. Thousands of papers had been published aimed at studying properties of systems by means of control, identification and other cybernetical means. It is important that overwhelming part of those papers were published in physical journals while their authors represent physical departments of the universities. The above facts provide evidences for existence of the new emerging field of research related both to physics and to control that can be called Cybernetical Physics 12, 13. A concise survey of cybernetical physics is presented in 14.

In this paper the possibilities of analyzing physical systems by means of feedback design are discussed. Firstly, the subject and methodology of cybernetical physics are outlined. Secondly, examples of transformation laws describing the excitability properties of dissipative systems are presented.

\section{Subject and Methodology of Cybernetical Physics}

Cybernetical Physics (CP) can be defined as the branch of science aimed at studying physical systems by cybernetical means. The problems constituting the subject of CP include control, identification, estimation and others. Due to limited size of the paper only the main class - control problems will be discussed. In order to characterize control problems related to $\mathrm{CP}$ one needs to specify classes of controlled plant models, control objectives (goals) and admissible control algorithms. Speaking about the methodology of CP, one needs to classify main methods uses for solving the problems and characterize typical results in the field. A brief description of the subject and methodology of CP is presented below.

\subsection{Models of Controlled Systems}

A formal statement of any control problem begins with a model of the system to be controlled (plant) and a model of the control objective (goal). Even if 
the plant model is not given (like in many real world applications) it should be determined in some way. The system models used in cybernetics are similar to traditional models of physics and mechanics with the only difference: the inputs and outputs of the model should be explicitly specified. The following main classes of models are considered in the literature related to control of physical systems. The most common class consists of continuous systems with lumped parameters described in state space by differential equations

$$
\dot{x}=F(x, u),
$$

where $x$ is $n$-dimensional vector of the state variables; $\dot{x}=d / d t$ stands for the time derivative of $x$; $u$ is $m$-dimensional vector of inputs (control variables). Vector-function $F(x, u)$ is usually assumed continuously differentiable to guarantee existence and uniqueness of the solutions of (2.1) at least at some time interval close to the initial point $t=0$. It is important to note that model (2.1) encompasses two physically different cases:

A. Coordinate control. The input variables represent some physical variables (forces, torques, intensity of electrical or magnetic fields, etc.) For example a model of a controlled oscillator (pendulum) can be put into the form

$$
J \ddot{\varphi}+r \dot{\varphi}+m g l \sin \varphi=u,
$$

where $\varphi=\varphi(t)$ is the angle of deflection from vertical; $J, m, l, r$ are physical parameters of the pendulum (inertia moment $J=m l^{2} / 2$, mass, length, friction coefficient); $g$ is gravity acceleration; $u=u(t)$ is the controlling torque. The description (2.2) is transformable into the form (2.1) with the state vector $x=$ $(\varphi, \dot{\varphi})^{\mathrm{T}}$.

B. Parametric control. The input variables represent change of physical parameters of the system, i.e. $u(t)=p-p_{0}$, where $p_{0}$ is the nominal value of the physical parameter $p$. For example, let the pendulum be controlled by changing its length: $l(t)=l_{0}+u(t)$. If $l(t)$ is slowly varying variable, then the model, instead of (2.2) becomes

$$
J \ddot{\varphi}+r \dot{\varphi}+m\left(l_{0}+u(t)\right) \sin \varphi=0
$$

If the rate of the length change $i(t)$ cannot be neglected, it is natural to choose it as the controlling variable:

$$
i(t)=u(t) .
$$

In this case the dynamics model derived from Euler-Lagrange equation instead of (2.3) takes the form

$$
m\left(l_{0}+u(t)\right)^{2} \ddot{\varphi}+2 m\left(l_{0}+u(t)\right) u(t) \varphi+r \dot{\varphi}+m g\left(l_{0}+u(t)\right) \sin \varphi=0
$$

and the plant model is described by equations (2.4), (2.5). 
Although in some papers the difference between the cases A and B is emphasized, for the purpose of studying the nonlinear system (2.1) the difference is not very important. 1

If external disturbances are present, we need to consider more general timevarying models

$$
\dot{x}=F(x, u, t) \text {. }
$$

On the other hand, many nonlinear control problems can be described using more simple affine in control models

$$
\dot{x}=f(x)+g(x) u .
$$

The model should also include the description of measurements, i.e. the $l$-dimensional vector of output variables (observables) $y$ should be defined, for example

$$
y=h(x) \text {. }
$$

An important example of output for physical systems is energy. E.g. for the pendulum (2.2) the energy is defined as follows: $H=0.5 J(\dot{\varphi})^{2}+m g l(1-\cos \varphi)$. Therefore it is not sufficient to consider only linear functions $h(x)$ as it is accustomed in control theory.

For many systems discrete-time state-space models are used

$$
x_{k+1}=F_{d}\left(x_{k}, u_{k}\right)
$$

where $x_{k} \in \mathbb{R}^{n}, u_{k} \in \mathbb{R}^{m}, y_{k} \in \mathbb{R}^{l}$, are state, input and output vectors at $k$ th stage of the process. Then the model will be defined by the mapping $F_{d}$. A lot of publications are devoted to control of distributed systems: delay-differential and delay-difference models, spatio-temporal systems described by partial differential equations or their discrete analogs, etc.

\subsection{Control Goals}

It is natural to classify control problems by their control goals. The conventional control goals are regulation and tracking. State tracking is driving a solution $x(t)$ of (2.1) to the prespecified desired function $x_{*}(t)$ i.e. fulfillment of the relation

$$
\lim _{t \rightarrow \infty}\left[x(t)-x_{*}(t)\right]=0
$$

for any solution $x(t)$ of (2.1) with initial conditions $x(0)=x_{0} \in \Omega$, where $\Omega$ is given set of initial conditions. Similarly, output tracking is driving the output $y(t)$ to the desired output function $y_{*}(t)$, i.e.

$$
\lim _{t \rightarrow \infty}\left[y(t)-y_{*}(t)\right]=0 .
$$

\footnotetext{
${ }^{1}$ It makes sense to treat differently the case of coordinate control and the case of parametric control for linear systems because the linear system with linear parametric feedback control leaves the class of linear systems (becomes bilinear). However the class of nonlinear systems (2.1) is closed with respect to all nonlinear feedbacks.
} 
The problem is to find a control function in the form of open loop (feedforward) control

$$
u(t)=U\left(t, x_{0}\right)
$$

in the form of state feedback

$$
u(t)=U(x(t))
$$

or in the form ofoutput feedback

$$
u(t)=U(y(t))
$$

to ensure the goal (2.10) or (2.11).

The key feature of the control problems for physical systems is that the goal should be achieved by means of sufficiently small (ideally, arbitrarily small) control. Solvability of this task is not obvious if the trajectory $x_{*}(t)$ is unstable, like for the case of chaotic systems, see [10.

A special case of the above problems is stabilization of the unstable equilibrium $x_{* 0}$ of system (2.1) with $u=0$, i.e. stabilization of $x_{* 0}$, satisfying $F\left(x_{* 0}, 0\right)=0$. Again, it looks like a standard regulation problem with an additional restriction that we seek for "small control" solutions. However, such a restriction makes the problem far from standard: even for a simple pendulum, nonlocal solutions of the stabilization problem with small control were obtained only recently, see [19]. The class of admissible control laws can be extended by introducing dynamic feedback described by differential or time-delayed models. Similar formulations hold for discrete and time-delayed systems.

Second class of control goals corresponds to the problems of excitation or generation of oscillations. The goal trajectory $x_{*}(t)$ is not necessarily periodic. Moreover, the goal trajectory may be specified only partially. In these cases a scalar goal function $G(x)$ is given and the goal is to achieve the limit equality

$$
\lim _{t \rightarrow \infty} G(x(t))=G_{*}
$$

or inequality

$$
\underline{\lim }_{t \rightarrow \infty} G(x(t)) \geq G_{*} .
$$

In many cases the total energy of mechanical or electrical oscillations can serve as $G(x)$.

Third important class of control goals corresponds to synchronization (more accurately, controlled synchronization as distinct from autosynchronization or self-synchronization. Generally speaking, synchronization is understood as concordance or concurrent change of the states of two or more systems or, perhaps, concurrent change of some quantities related to the systems, e.g. equalizing of oscillation frequencies. If the required relation is established only asymptotically, one speaks about asymptotic synchronization. If synchronization does not exist in the system without control (for $u=0$ ) we may pose the problem as finding the control function which ensures synchronization in the closed-loop system, i.e. synchronization may be a control goal. For example the goal corresponding to 
asymptotic synchronization of the two system states $x_{1}$ and $x_{2}$ can be expressed as follows:

$$
\lim _{t \rightarrow \infty}\left[x_{1}(t)-x_{2}(t)\right]=0
$$

In the extended state space $x=\left\{x_{1}, x_{2}\right\}$ of the overall system, relation (2.17) implies convergence of the solution $x(t)$ to the diagonal set $\left\{x: x_{1}=x_{2}\right\}$. Asymptotic identity of the values of some quantity $G(x)$ for two systems can be formulated as

$$
\lim _{t \rightarrow \infty}\left[G\left(x_{1}(t)\right)-G\left(x_{2}(t)\right)\right]=0 .
$$

Often it is convenient to rewrite the goals (2.10), (2.11), (2.15), (2.17) or (2.18) in terms of appropriate goal function $Q(x, t)$ as follows:

$$
\lim _{t \rightarrow \infty} Q(x(t), t)=0 .
$$

For example to reduce goal (2.17) to the form (2.19) one may choose $Q(x)=\mid x_{1}-$ $\left.x_{2}\right|^{2}$. Instead of Euclidean norm other quadratic functions can also be used. E.g. for the case of the goal (2.10) the goal function $Q(x, t)=\left[x-x_{*}(t)\right]^{\mathrm{T}} \Gamma\left[x-x_{*}(t)\right]$, where $\Gamma$ is positive definite symmetric matrix can be used. The freedom of choice of the goal function can be utilized for design purposes.

Finally, the goals may be specified as modification of some quantitative requirements to the limit behavior of the system, i.e. changing fractal dimension of its attractor.

Some of the above mentioned goals are not typical for conventional control theory because they do not specify the desired behavior of the system completely. These classes of control problems belong to the area of the so called partial control which development has become active recently 15, 16. It is important that the above goals should be achieved without significant intervening the system dynamics, i.e. the control algorithms to be designed should meet the small control or weak control requirement. Therefore typical formulations of the control problems look as follows:

- find all the behaviors that can be attained by the control functions of the given (sufficiently small) norm;

- find on control function (feedback operator) of minimum norm ensuring the given control goal.

Of course, traditional formulations are not ruled out and may appear in some physical problems.

\subsection{Methodology}

The methodology of cybernetical physics is based on the control theory. Typically, some parameters of physical systems are unknown and some variables are not available for measurement. From the control viewpoint it means that control design should be performed under significant uncertainty, i.e. methods of robust or adaptive control should be used. A variety of design methods have been developed both for linear and for nonlinear systems [16,17, 18. Methods of partial control and weak control are also available [11,15. 
Speed-Gradient Method. Let us describe a fairly general approach to control algorithms design for nonlinear systems: the so called speed-gradient method. It is intended for control of continuous-time systems with the control goal specified by means of a goal function. Consider a nonlinear time-varying system and control goal (2.19), where $Q(x, t) \geq 0$ - is a smooth goal function.

In order to design control algorithm the scalar function $\dot{Q}=\omega(x, u, t)$ is calculated that is the speed (rate) of changing $Q_{t}=Q(x(t), t)$ along trajectories of (2.6) $: \omega(x, u, t)=\partial Q(x, t) / \partial t+\left[\nabla_{x} Q(x, t)\right]^{\mathrm{T}} F(x, u, t)$. Then evaluate the gradient of $\omega(x, u, t)$ with respect to input variables $\nabla_{u} \omega(x, u, t)=(\partial \omega / \partial u)^{\mathrm{T}}=$ $(\partial F / \partial u)^{\mathrm{T}} \nabla_{x} Q(x, t)$. Finally, the algorithm of changing $u(t)$ is determined according to the differential equation

$$
\frac{d u}{d t}=-\Gamma \nabla_{u} \omega(x, u, t)
$$

where $\Gamma=\Gamma^{\mathrm{T}}>0$ is a positive definite gain matrix, e.g. $\Gamma=\operatorname{diag}\left\{\gamma_{1}, \ldots, \gamma_{m}\right\}$, $\gamma_{i}>0$. The algorithm (2.20) is called speed-gradient (SG) algorithm, since it suggests to change $u(t)$ proportionaly to the gradient of the speed of changing $Q_{t}$.

The origin of the algorithm (2.20) can be explained as follows. In order to achieve the control goal (2.19) it is desirable to change $u(t)$ in the direction where $Q(x(t), t)$ decrease. However it may be a problem since $Q(x(t), t)$ does not depend on $u(t)$ directly. Instead one may try to decrease $\dot{Q}$, in order to achieve the inequality $\dot{Q}<0$, which implies decrease of $Q(x(t), t)$. The speed $\dot{Q}=\omega(x, u, t)$ generically depends on $u$ explicitly which allows to write down (2.20). The speed-gradient algorithm can be also interpreted as a continuoustime counterpart of the gradient algorithm, since for small sampling step size the direction of the gradient is close to the direction of the speed-gradient.

For special case of the system linear in inputs the algorithm (2.20) is nothing but a classical integral control law.

Similarly the following SG-algorithm in finite form is introduced which is a generalization of a proportional control law:

$$
u(t)=u_{0}-\Gamma \nabla_{u} \omega(x(t), u(t), t),
$$

where $u_{0}$ is some initial value of control variable, e.g. $\left.u_{0}=0\right)$. More general form of the SG-algorithms can also be used:

$$
u(t)=u_{0}-\gamma \psi(x(t), u(t), t),
$$

where $\gamma>0$ is the scalar gain parameter and vector-function $\psi(x, u, t)$ satisfies the so called pseudogradient condition

$$
\psi(x, u, t)^{\mathrm{T}} \nabla_{u} \omega(x, u, t) \geq 0 .
$$

Special cases of (2.22) are sign-like and relay-like algorithms

$$
u(t)=u_{0}-\gamma \operatorname{sign} \nabla_{u} \omega(x(t), u(t), t),
$$


where (sign) of a vector is understood component-wise: for a vector $x=\operatorname{col}\left(x_{1}\right.$, $\left.\ldots, x_{m}\right)$ is defined as $\operatorname{sign} x=\operatorname{col}\left(\operatorname{sign} x_{1}, \ldots, \operatorname{sign} x_{m}\right)$.

In order to make a reasonable choice of the control algorithm parameters the applicability conditions should be developed and checked. A number of applicability conditions can be found in 11,16]. The main conditions are the following ones: convexity of the function $\omega(x, u, t)$ in $u$ and existence of "ideal" control $u_{*}$ such that $\omega\left(x, u_{*}, t\right) \leq 0$ for all $x$ (attainability condition).

The speed-gradient algorithm is tightly associated to the concept of Lyapunov function $V(x)$ - a function of the system state nonincreasing along its trajectories. Lyapunov function is an abstraction for such physical characteristics like energy and entropy. I is important that Lyapunov function can be used not only for analysis but also for system design. In particular, the speed-gradient algorithms in the finite form have Lyapunov function the goal function itself: $V(x)=Q(x)$, while differential form of SG-algorithms corresponds to the Lyapunov function of the form: $V(x, u)=Q(x)+0.5\left(u-u_{*}\right)^{\mathrm{T}} \Gamma^{-1}\left(u-u_{*}\right)$, where $u_{*}-$ is the desired "ideal" value of controlling variables.

\subsection{Results: Laws of Cybernetical Physics}

A great deal of the results in many areas of physics are presented in form of conservation laws, stating that some quantities do not change during evolution of the system. However, the formulations in CP are different. The results in CP establish how the evolution of the system can be changed by control. Therefore the results in CP should be formulated as transformation laws, specifying the classes of changes in the evolution of the system attainable by control function from the given class, i.e. specifying the limits of control. Typical example of transformation law: "Any controlled chaotic trajectory can be transformed into a periodic one by means of control" (OGY law 10]). Another example related to excitation of a system with small control will be presented below.

The term "controllable" in the above context means principal solvability of the problem. To apply the law one needs to use some sufficient conditions ensuring controllability which, however are a matter of further mathematical investigation.

\section{Examples of Transformation Laws. Excitability}

Many complex systems, including complex networks possess some characteristics that are invariant or decreasing along the trajectories of the nominal (unforced, undisturbed) system. Such functions are analogs of energy for physical (e.g. mechanical) systems and play a important role in system analysis and design. For example, it may be a total utility function in the problems of internet congestion control [23] or energy-like function evaluating the failure threshold [22. Sometimes a network can be endowed with a generalized Hamiltonian structure which significantly reduces complexity of its analysis 22. The goal of control in such cases may be to stabilize or to increase the value of the above characteristics and important question is the one about the limitations of such a control. Below 
the limits of energy control for Hamiltonian and dissipative systems are established which provide an example of transformation laws in nonlinear systems.

\subsection{Measuring Excitability of Systems}

In 12,13, the idea to create maximum excitation (resonance) mode in a nonlinear system by changing the frequency of external action as a function of oscillation amplitude was suggested. The corresponding phenomenon in system behavior was termed "feedback resonance". Such a sort of control may be used in a broader class of problems when the goal is to maximize or to minimize the limit value of some energy-like characteristic of a complex system under bounded power of control action.

Consider a system described by state-space equations

$$
\dot{x}=F(x, u), y=h(x),
$$

where $x \in R^{n}$ is state vector, $u, y$ are scalar input and output, respectively.

To realize the idea of feedback resonance, $u(t)$ should depend on the state of the system $x(t)$ or on the current measurements $y(t)$, which exactly means introducing a state feedback $u(t)=U(x(t))$ or output feedback $u(t)=U(x(t))$. Now the problem is: how to find the feedback law in order to achieve the maximum limit amplitude of output?

Let us pose the problem as that of optimal control: to find

$$
\begin{gathered}
Q(\gamma)=\limsup |y(t)|^{2} . \\
|u(s)| \leq \gamma, \\
0 \leq s \leq t \\
x(0)=0 \\
t \geq 0
\end{gathered}
$$

Assume that the system (3.25) is BIBO stable (bounded input produce bounded output) and $x=0$ is equilibrium of the unforced system $(F(0,0)=0, h(0)=0)$ in order to ensure $Q(\gamma)$ to be well defined. Apparently, the signal providing maximum excitation should depend not only on time but also on system state, i.e. input signal should have a feedback form. Note that for linear systems the value of the problem (3.26) depends quadratically on $\gamma$. Therefore it is naturally to introduce the excitability index (EI) for the system (3.25) as follows:

$$
E(\gamma)=\frac{1}{\gamma} \sqrt{Q(\gamma)}
$$

where $Q(\gamma)$ is the optimum value of the problem (3.26). It is clear that for linear asymptotically stable systems $E(\gamma)=$ const. For nonlinear systems $E(\gamma)$ is a function of $\gamma$ that characterizes excitability properties of the nonlinear system. It was introduced in [14] with respect to the energy-like output. For MIMO systems excitability indices $E_{i j}$ can be introduced in a similar way for every pair of input $u_{i}$ and output $y_{j}$. The concept of EI is related to the concept of input-output (I-O) gain If I-O gain exists, it provides an upper bound for EI. Conversely, if $\mathrm{EI}$ is finite, it estimates the minimal value of I-O gain. 
The solution to the problem (3.26) for nonlinear systems is quite complicated in most cases. However we can use approximate locally optimal or speed-gradient solution

$$
u(x)=\gamma \operatorname{sign}\left(g(x)^{\mathrm{T}} \nabla h(x) h(x)\right),
$$

where $g(x)=\left.\frac{\partial F(x, u)}{\partial u}\right|_{u=0}$, obtained by maximizing the principal part of instant growth rate of $|y(t)|^{2}$. It follows from the results of 20] that for small $\gamma$ the value of $|y(t)|$ achievable with input (3.28) for sufficiently large $t \geq 0$ differs from the optimal value $Q(\gamma)$ by the amount of order $\gamma^{2}$. An important consequence is that excitability index can be estimated directly by applying input (3.28) to the system. For real world systems it can be done experimentally. Otherwise, if a system model is available, computer simulations can be used.

\subsection{Properties of Excitability Index}

Since excitability index of a system characterizes its sensitivity to a feedback excitation, it is important to relate excitability to inherent dynamical properties of a system. Such kind of bounds for a class of strictly passive systems (systems with full dissipation) were established in 14. We present here a slightly modified result.

Recall that the system (3.25) is called strictly passive with dissipation rate $\rho(x) \geq 0$ if there exists continuous nonnegative function $V(x)$ (storage function) such that for all $t \geq 0$ and any solution $x(t)$ of the system (3.25) the following identity holds

$$
V(x(t))=V(x(0))+\int_{0}^{t}\left(w(s)^{\mathrm{T}} u(s)-\varrho(x(s))\right) d s,
$$

where $w=W(x)$ is an auxiliary output function of the system, the so called passivity output.

The storage function $V(x)$ is an analog of energy for the systems of general form (3.25), i.e. identity (3.29) can be interpreted as the generalized energy balance.

Definition 1. Let the set of admissible control consist of functions $u(t)$, bounded for $0 \leq t<\infty$ such that the corresponding trajectories $x(t)$ are bounded. Define upper and lower excitability indices of (3.25) with respect to the output $V(x)$ as functions $\chi_{V}^{+}(\gamma), \chi_{V}^{-}(\gamma)$, defined for $0 \leq \gamma<\infty$ as follows:

$$
\begin{aligned}
& \chi_{V}^{+}(\gamma)=\varlimsup_{t \rightarrow \infty} \sup _{\substack{|u(\cdot)| \leq \gamma \\
x(0)=0}} V(x(t)), \\
& \chi_{V}^{-}(\gamma)=\lim _{\overline{t \rightarrow \infty}} \sup _{\substack{|u(\cdot)| \leq \gamma \\
x(0)=0}} V(x(t)) .
\end{aligned}
$$

Similarly excitability indices $\chi_{y}^{+}(\gamma), \chi_{y}^{-}(\gamma)$ with respect to any output $y=$ $h(x)$ are defined. In the case when the input is vector, $u=\operatorname{col}\left\{u_{1}, \ldots, u_{m}\right\}$ 
output is also vector $\gamma=\left\{\gamma_{1}, \ldots, \gamma_{m}\right\}$ and excitability indices are defined as multi-indices. In general case of the system with $m$ inputs and $l$ outputs the excitability indices $\chi_{y}^{+}(\gamma), \chi_{y}^{-}(\gamma)$ are $l \times m$-matrices depending on $m$ arguments. Note that the technical assumption of boundedness of $x(t)$ can be weakened but we will not do it here for simplicity.

The main result of this section is the following statement.

Theorem 1. Let system (3.25) be strictly passive and the storage function $V(x)$ and dissipation rate $\varrho(x)$ satisfy inequalities

$$
\begin{gathered}
\alpha_{0}|w|^{2} \leq V(x) \leq \alpha_{1}|w|^{2}+d, \\
\varrho_{0}|w|^{2} \leq \varrho(x) \leq \varrho_{1}|w|^{2}
\end{gathered}
$$

for some positive $\alpha_{0}, \alpha_{1}, \varrho_{0}, \varrho_{1}, d$. Let the set

$$
\Omega^{-}=\left\{x: W(x)=0, V(x)<\alpha_{0}\left(\frac{\gamma}{\varrho_{1}}\right)^{2}\right\}
$$

not contain whole trajectories of the free system $\dot{x}=F(x, 0)$.

Then excitability indices $\chi_{V}^{+}(\gamma), \chi_{V}^{-}(\gamma)$ with respect to $V(x)$ satisfy inequalities

$$
\alpha_{0}\left(\frac{\gamma}{\varrho_{1}}\right)^{2} \leq \chi_{V}^{-}(\gamma) \leq \chi_{V}^{+}(\gamma) \leq m \alpha_{1}\left(\frac{\gamma}{\varrho_{0}}\right)^{2}+d,
$$

Besides, the lower bound is realized for the speed-gradient control

$$
u(t)=\gamma \operatorname{sign} w(t)
$$

We see that the action (3.28) or (3.35) creates a sort of resonance mode in a nonlinear system: for weakly damped systems even a small action having form (3.28) leads to large oscillations of the output and can insert a substantial amount of energy into the system. Relations (3.34) can be interpreted as transformation of energy laws for passive systems:

In a strictly passive system with small dissipation of order $\rho$ an energy level achievable by means of control of the level not exceeding $\gamma$ is of $\operatorname{order}(\gamma / \rho)^{2}$.

\subsection{Case of Hamiltonian Systems. Escape from Potential Wells}

The above limits for excitability of physical systems by means of controlling actions are expressed in terms of the ratio "(excitation amplitude)/(dissipation)". They can be applied to systems described by Hamiltonian models with possible dissipation:

$$
\dot{q}_{i}=\frac{\partial H(q, p, u)}{\partial p_{i}}, \quad \dot{p}_{i}=-\frac{\partial H(q, p, u)}{\partial q_{i}}-R_{i}(q, p), \quad i=1, \ldots, n,
$$


where $q=\operatorname{col}\left(q_{1}, \ldots, q_{n}\right), p=\operatorname{col}\left(p_{1}, \ldots, p_{n}\right)$ are vectors of generalized coordinates and momenta which form the system state vector $x=\operatorname{col}(q, p) ; H=$ $H(q, p, u)$ is the Hamiltonian function for controlled system; $u(t) \in \mathbb{R}^{m}$ is controlling input function; $R(q, p)=\operatorname{col}\left(R_{1}(q, p), \ldots, R_{n}(q, p)\right)$ is the dissipation function, satisfying the inequality

$$
R(q, p)^{\mathrm{T}} \frac{\partial H_{0}(q, p)}{\partial p} \geq 0,
$$

where $H_{0}(q, p)=H(q, p, 0)$ is the energy of free system. In what follows we assume that the Hamiltonian is linear in control: $H(p, q, u)=H_{0}(p, q)+H_{1}(p, q)^{\mathrm{T}} u$, where $H_{0}(p, q)$ is the internal Hamiltonian and $H_{1}(p, q)$ is an $m$-dimensional vector of interaction Hamiltonians.

Hamiltonian description is typical for many physical systems, including molecular systems. There are also attempts to apply Hamiltonian models for communication networks 22. The inequality (3.37) means dissipation of the energy over trajectories of the free (uncontrolled) system: $\dot{H}_{0} \leq 0$. It means that systems (3.36) are passive in the sense of (3.29) and Hamiltonian of the free system can be chosen as a storage function: $V(x)=H_{0}(q, p)$, while passivity output is the Poisson bracket $W(x)=\left[H_{0}, H_{1}\right]$ of smooth functions $H_{0}(p, q)$ and $H_{1}(p, q)$ defined in a standard manner:

$$
\left[H_{0}, H_{1}\right]=\sum_{i=1}^{n}\left(\frac{\partial H_{0}}{\partial p_{i}} \frac{\partial H_{1}}{\partial q_{i}}-\frac{\partial H_{0}}{\partial q_{i}} \frac{\partial H_{1}}{\partial p_{i}}\right) .
$$

As an example consider the problem of escape from a potential well which is important in many fields of physics and mechanics. Sometimes escape is an undesirable event and it is important to find conditions preventing it (e.g. buckling of the shells, capsize of the ships, etc.). In other cases escape is useful and the conditions guaranteeing it are needed. Escape may correspond to a phase transition in the system. In the area of information physics briefly described in the Introduction, escape may correspond to transition from the state " 0 " to state " 1 " of the information system, i.e. to creation of a bit of information. In all cases the conditions of achieving escape by means of as small external force as possible are of interest.

Consider nonlinear oscillators with one degree of freedom, modeled as

$$
\ddot{\varphi}+\varrho \dot{\varphi}+\Pi(\varphi)^{\prime}=u
$$

where $\varrho>0$ is the damping coefficient. Equation (3.38) can be transformed to the Hamiltonian form with coordinate and momentum $q=\varphi, p=\dot{\varphi}$, the Hamiltonian function (energy) $H_{0}(\varphi, \dot{\varphi})=\frac{1}{2} \dot{\varphi}^{2}+\Pi(\varphi)$ and passivity output $p$.

In 21] such a possibility (optimal escape) has been studied for typical nonlinear oscillators with a single-well potential $\Pi_{e}(\varphi)=\varphi^{2} / 2-\varphi^{3} / 3$ (so called "escape equation") and a twin-well potential $\Pi_{D}(\varphi)=-\varphi^{2} / 2+\varphi^{4} / 4$ (Duffing 
oscillator). The least amplitude of a harmonic external forcing $u(t)=\bar{u} \sin \omega t$ for which no stable steady state motion exists within the well was determined by intensive computer simulations. For example, for escape equation with $\varrho=0.1$ the optimal amplitude was evaluated as $\bar{u} \approx 0.09$, while for Duffing twin-well equation with $\varrho=0.25$ the value of amplitude was about $\bar{u} \approx 0.21$.

Using feedback forcing we may expect reducing the escape amplitude. In fact using the locally optimal control

$$
u=\gamma \operatorname{sign}(\dot{q})
$$

the amplitude of feedback leading to escape can be easily calculated, just substituting the height of potential barrier $\max _{\Omega} \Pi(\varphi)-\min _{\Omega} \Pi(\varphi)$ for $\bar{H}$ into (3.34) where $\Omega$ is the well corresponding to the initial state, see 12 . For example, in the case of escape equation $\bar{H}=1 / 6, \varrho=0.1$ and $\bar{u}=0.0577$, while for Duffing oscillator with $\bar{H}=1 / 4, \varrho=0.25$ escape amplitude is estimated as $\bar{u}=0.1767$. The obtained values are substantially smaller than those evaluated in [21]. The less the damping, the bigger the difference between the amplitudes of feedback and nonfeedback signals leading to escape. Simulation exhibits still stronger difference: escape for Duffing oscillator occurs for $\bar{u}=\gamma=0.122$ if the feedback (3.39) is applied. Note that the oscillations in the feedback systems have both variable frequency and variable shape.

In 12 the dependence of escape amplitudes on the damping was also studied by means of computer simulations in the range of damping coefficient $\varrho$ varying from 0.01 to 0.25 . Simulations confirmed theoretical conclusion that the feedback input amplitude required for escape is proportional to the damping. We may evaluate the efficiency of feedback $\mu$ as the ratio of escape amplitudes for harmonic $\left(\bar{u}_{h}\right)$ and feedback $\left(\bar{u}_{f}\right)$ forcing: $\mu=\bar{u}_{h} / \bar{u}_{f}$. Then the efficiency of feedback is inversely proportional to the damping for small values of damping.

\section{Conclusions}

Now, after a decade of vigorous development, cybernetical physics is still an emerging field. In the paper the limits for the speed-gradient control of energylike characteristics have been demonstrated providing an example of transformation law for dissipative systems.

The above mentioned results provide also a good starting point to apply speed-gradient method to control of failures in complex networks based on generalized Hamiltonian model of [22. Speed-gradient control of escape from potential wells may be helpful to control of molecular objectives, necessary to achieve physical limits of information transmission.

An interest of control community to control problems for communication networks has increased during recent years significantly [23, 24, 25]. Other 
existing methods of nonlinear control are under further development to become applicable to new important problems.

\section{References}

1. Landauer, R. The physical nature of information. Phys. Lett. A. 217 (1996) 188193.

2. Shannon, C. A Mathematical Theory of Communication. Bell Syst. Tech. J. 27 (1948) 3, 379-423; 4, 623-656.

3. von Neumann, J. Theory of Self-Reproducing Automata, edited and completed by Arthur W. Burks, University Illinois Press, Urbana and London (1966).

4. Landauer, R. Irreversibility and heat generation in the computing process. IBM J. Res. Develop 3 (1961) 183191.

5. Landauer, R. Energy Requirements in Communication. Appl. Phys. Lett. 51 (1987) 2056-2058.

6. Landauer, R. Minimal Energy Requirements in Communication, Science 272 (1996) 1914-1918.

7. John D. Norton, Eaters of the Lotus: Landauer's Principle and the Return of Maxwell's Demon", PHIL-SCI 1729 http://philsciarchive.pitt.edu/archive/00001729/

8. Bartsev S.I., Okhonin V.A. Adaptive networks of information processing. Krasnoyarsk, Inst. of Physics SO AN SSSR, 1986 (In Russian).

9. Brockett R.W. Control theory and analytical mechanics. Geometric Control Theory, Lie Groups. V. VII / Eds. C. Martin, R. Hermann. Mat. Sci. Press, Brookine, MA, (1977) 1-48.

10. Ott T., Grebogi C., Yorke G. Controlling chaos. Phys. Rev. Lett. 64 (1990) 11 1196-1199.

11. Fradkov A.L., Pogromsky A.Yu. Introduction to control of oscillations and chaos. World Scientific, Singapore (1998).

12. Fradkov A.L. Exploring nonlinearity by feedback. Physica D. 128 (1999) No 2-4 159-168.

13. Fradkov A.L. Investigation of physical systems by means of feedback. Autom. Remote Control 60 (1999) 3 3-22.

14. Fradkov A.L. Cybernetical physics. Nauka, St.Petersburg 2003, 208p.(In Russian).

15. Vorotnikov V.I. Partial Stability and Control. Birkhäuser (1998).

16. Fradkov A.l., Miroshnik I.V., Nikiforov V.O. Nonlinear and adaptive control of complex systems. Dordrecht: Kluwer Academic Publishers (1999).

17. Krstić, M., I. Kanellakopoulos and P.V. Kokotović. Nonlinear and Adaptive Control Design, New York, Wiley (1995).

18. Zhou K., J.C.Doyle and K.Glover. Robust and Optimal Control. Prentice Hall (1996).

19. Shiriaev A.S. and A.L.Fradkov. Stabilization of invariant sets for nonlinear systems with application to control of oscillations. Intern. J. of Robust and Nonlinear Control, 11 (2001) 215-240.

20. Chernousko F.L. Some problems of optimal control with a small parameter. J. Appl. Math. Mech. (1968) 3 12-22.

21. Stewart, H.B., Thompson, J.M.T., Ueda, U. \& Lansbury, A.N. Optimal escape from potential wells - patterns of regular and chaotic bifurcations. Physica D $\mathbf{8 5}$ (1995) 259-295. 
22. DeMarco C.L. A phase transition model for cascading network failure. IEEE Control Systems Magazine, 21 (2001) 6 40-51.

23. Law S., Paganini F., Doyle J. Internet congestion control. IEEE Control Systems Magazine, 22 (2002) 1 28-43.

24. Wen, J.T., Arcak M. A unifying passivity framework for network flow control. IEEE Trans. Autom. Contr. AC-49 (2004) 2 162-174.

25. Special Issue on Networked Control Systems. Eds. P.Antsaklis and J.Baillieul. IEEE Trans. Autom. Contr. AC-49 (2004) 9. 